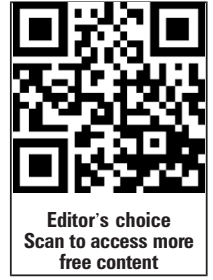

10.1136/eb-2012-101046

Department of Paediatric Pulmonology, Wilhelmina Children's Hospital, University Medical Centre Utrecht, Utrecht, The Netherlands

Correspondence to: Cornelis K van der Ent Department of Paediatric Pulmonology, Wilhelmina Children's Hospital, University Medical Center Utrecht, Lundlaan 6, Utrecht 3508 AB, The Netherlands; K.vanderent@umcutrecht.nl

\title{
Contact with dogs during the first year of life is associated with decreased risk of respiratory illness
}

\section{Cornelis K van der Ent, Anne C van der Gugten}

Commentary on: Bergroth E, Remes S, Pekkanen J, et al. Respiratory tract illnesses during the first year of life: effect of dog and cat contacts. Pediatrics 2012;130:211-20.

\section{Implications for practice and research}

- Early dog contacts are associated with fewer respiratory infections in infants, especially otitis, but not wheezing after adjustment for possible confounders.

- Early animal contacts are important, possibly leading to changes in immune development and a better resistance to infectious respiratory diseases in infants.

- Future research is needed to explore the mechanism of the favourable effect of dog exposure and whether similar results occur over a longer period.

\section{Context}

Several groups have studied the associations between animal contacts early in life and asthma and allergic diseases in childhood. ${ }^{1}$ Reports on the association between pet exposure and respiratory infection are sparse. Results have been inconsistent, due to retrospective evaluation, study of children of different age groups and having no separate analysis of different pets. Bergroth and colleagues explored the association between $\operatorname{dog}$ and cat contacts during infancy and respiratory illnesses during the first year of life by using weekly diaries filled in by the parents.

\section{Methods}

Two prospective cohorts of Finnish infants, born between September 2002 and May 2005, were included in the study. A total of 208 children from the PASTURE birth cohort study were studied, whose mothers (all living in rural areas) were followed up from the third trimester of pregnancy. Another 216 mothers and infants living in either rural or suburban environments were also included. From the 9th to the 52nd week of age of their child, all parents were asked to fill in weekly diary logs, concerning different infectious diseases and symptoms. Detailed information about cats and dogs in the house and the time a child spent in the house was recorded. Potential confounding variables like parental atopy, number of siblings, living environment and day-care attendance were obtained. Children, for whom less than half of the questionnaires were filled in, were excluded from data analysis.

\section{Findings}

Children with dog contacts at home were significantly healthier during the study period, had fewer weeks with fever and otitis, and needed fewer courses of antibiotics than children without dog contacts, after adjustment for potential confounders. The sensitivity analysis with children removed families reporting avoidance of pets, due to some allergic causes, showed the same results. No significant associations were found between cat contact and the different study outcomes.

\section{Commentary}

The results of this study showed that dog contact during early infancy may be associated with less morbidity in general and less upper respiratory tract infections. The authors speculate that dog contact might help to mature the immunological system, leading to more composed immunological response and shorter duration of infections.

It is important to realise that studies of animal exposure and respiratory symptoms can be easily influenced by selection bias, because the atopic status of parents or older siblings can determine whether a family keeps pets or not. Although adjustment for parental atopy, and removing children with a reported history of pet avoidance, did not alter the results, the influence of possible atopic predisposition cannot be completely ruled out. Exposure of the child to animals outside their own home, for example, outdoors, at the houses of relatives or at daycare was not reported, while this might significantly influence the total exposure of the child. Research suggests that pet owners are different from non-owners in terms of personality characteristics. ${ }^{2}$ Therefore, one could speculate that pet owners experience and report the symptoms of their child as less severe than non-owners which might have resulted in information bias.

More research is necessary to confirm the results of this study. In order to more fully understand the differential associations between dog-and-cat exposure and later respiratory outcome, the children should be followed for a longer period. Unravelling the mechanism of the favourable effects of dog exposure might help in preventing respiratory illnesses in children.

Funding None.

Competing interests CKE has received unrestricted grants from Grunenthal and GlaxoSmithKline.

\section{References}

1. Lodrup Carlsen KC, Roll S, Carlsen KH, et al. Does pet ownership in infancy lead to asthma or allergy at school age? Pooled analysis of individual participant data from 11 European birth cohorts. PLoS One 2012;7:e43214.

2. Flint EL, Minot EO, Perry PE, et al. Characteristics of adult dog owners in New Zealand. N Z Vet J 2010;58:69-73. 